\title{
Generation of Femtosecond Synchrotron Pulses: Performance and Characterization
}

\author{
R.W. Schoenlein ${ }^{\mathrm{a}}$, A. Cavalleri ${ }^{\mathrm{a}}$, H.H.W. Chong ${ }^{\mathrm{b}}$, T.E. Glover ${ }^{\mathrm{c}}$, \\ P.A. Heimann ${ }^{c}$, A.A. Zholents ${ }^{\mathrm{d}}$, M.S. Zolotorev ${ }^{\mathrm{d}}$ \\ ${ }^{a}$ Materials Sciences Division, Lawrence Berkeley National Laboratory, Berkeley, CA 94720, USA \\ ${ }^{b}$ Applied Sciences Graduate Program, U.C. Berkeley, Berkeley, CA 94720, USA \\ ${ }^{c}$ Advanced Light Source, Lawrence Berkeley National Laboratory, Berkeley, CA 94720, USA \\ ${ }^{d}$ Accelerator and Fusion Research Division, Lawrence Berkeley National Laboratory, Berkeley, CA 94720, USA
}

\begin{abstract}
Femtosecond synchrotron pulses of $<200$ fs duration are generated at the Advanced Light Source beamline 5.3.1 via laser manipulation of the stored electron beam. We demonstrate a peak laser acceleration of $>13 \mathrm{MeV}$ relative to the nominal $1.9 \mathrm{GeV}$ beam energy. Femtosecond pulses are effectively isolated from the long-pulse background using the transverse dispersion of the storage ring in combination with an x-ray imaging optic and a pair of slits to achieve a signal/background ratio of $\sim 1$.
\end{abstract}

\section{INTRODUCTION}

An important frontier in ultrafast research is the application of femtosecond $\mathrm{x}$-ray pulses to investigate structural dynamics associated with chemical reactions, phase transitions in solids, and rapid biological processes. The fundamental time scale for such processes is an atomic vibrational period, $\sim 100 \mathrm{fs}$, which is nearly three orders of magnitude beyond the present capabilities of synchrotrons. We have developed a novel technique for generating femtosecond x-ray pulses from the Advanced Light Source (ALS) using ultrashort laser pulses to manipulate the temporal and spatial structure of stored electron bunches ${ }^{1-3}$. As illustrated in Fig. 1, the electron beam is modulated via co-propagation with a femtosecond laser pulse in a resonantly-tuned undulator. Subsequently, the modulated femtosecond-duration electron bunch is spatially separated from the main bunch by means of the horizontal dispersion bump associated with the bend magnets in the first arc following the wiggler. Femtosecond x-rays are generated from the displaced electrons passing through the central bend magnet, and are imaged onto a pair of slits to discriminate against the long-pulse background $\mathrm{x}$-rays. The original electron bunch is recovered due to synchrotron damping of the electrons in the storage ring. Thus, other synchrotron beamlines are unaffected and special operation of the storage ring is not required.

In previous proof-of-principle experiments at ALS beamline 6.3.2, the time structure created on the electron bunch (through interaction with the femtosecond optical pulses) was measured via cross-correlation of the visible synchrotron radiation with a second (delayed) laser pulse ${ }^{2}$. Since the temporal structure of the incoherent synchrotron radiation is determined entirely by the temporal structure of the electron bunch, the generated femtosecond pulse duration will be invariant from the infrared to the x-ray range. However, in addition to the time structure, an important criterion for experimental applications is the extent to which the femtosecond $\mathrm{x}$-ray pulses can be isolated from the long-pulse background. In experimental applications using the pump-probe technique, the femtosecond $\mathrm{x}$-rays are used as a probe, and are detected using integrating detectors (e.g. avalanche photodiodes, or microchannel plates) that measure the flux over a single synchrotron pulse (modulated by the femtosecond laser) and yet have a sufficiently fast response to gate out the remaining synchrotron pulses.

The utility of the femtosecond x-ray pulses is ultimately determined by the extent to which they can be distinguished from the long-pulse background integrated over a single pulse. For shot-noise-limited detection, the data acquisition time required to achieve a given signal-to-noise ratio scales as $(1+1 / \mathrm{F})$ where $\mathrm{F}$ is the ratio of the femtosecond signal to the long-pulse background. This ratio, F, is determined by three factors: (i) the amplitude of 
the energy modulation, $\Delta \mathrm{E}$, created in the electron bunch by the laser pulse, (ii) the horizontal (dynamic) dispersion, $\mathrm{D}_{\mathrm{x}}$, in the region of the storage ring where the x-rays are generated, and (iii) the quality of the x-ray beam image at the slits, as determined by the transverse profile of the electron beam and the imaging optics in the beamline.

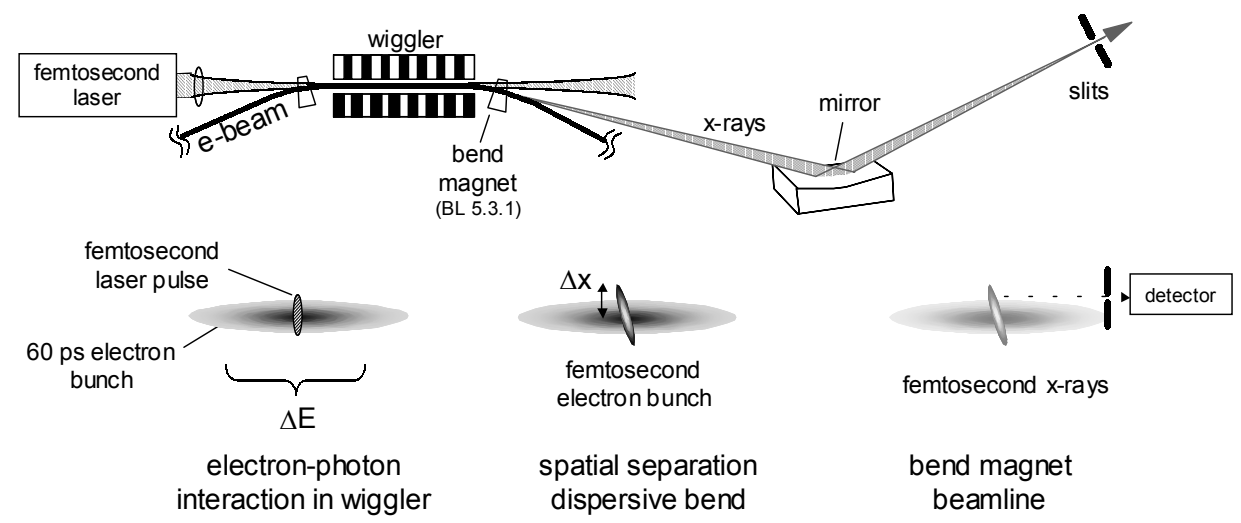

FIGURE 1. Schematic illustration of the method for generating femtosecond x-rays from a storage ring: A femtosecond laser pulse energy-modulates the electron bunch as they co-propagate through a resonantly-tuned wiggler. A dispersive section of the storage ring maps the energy modulation, $\Delta \mathrm{E}$, to a transverse spatial separation, $\Delta \mathrm{x}$, of a femtosecond slice of the electron bunch. $\mathrm{X}$-rays generated by the modulated electron bunch (in a bend-magnet) are collected and imaged onto a slit that is used to select the femtosecond x-rays (generated off axis).

\section{X-ray Beam Profile Measurements}

For experimental applications, we require that the long-pulse background (integrated over a single synchrotron pulse) be less than the integrated flux from the femtosecond $x$-ray pulse $(\mathrm{F}>1)$. The latter is determined by the ratio of the laser pulse duration to the synchrotron pulse duration (typically $10^{-3}$ ) and by the width of the slits relative to the total transverse displacement, $2 \Delta \mathrm{x}$, of the femtosecond electron slice (typically $\left.10^{-1}\right)^{3}$. Thus, the flux of the femtosecond $\mathrm{x}$-ray pulse is $\sim 10^{-4}$ of the total flux from a single bunch, and this establishes the required image quality of the x-ray beam at the slits.

The quality of the x-ray beam image is primarily determined by two factors, the transverse profile of the electron beam at the radiation point, and the imaging properties of the beamline optics. In the case of the electron beam, knife-edge measurements of the beam profile at the ALS indicate that it follows a Gaussian horizontal distribution out to $6 \sigma_{\mathrm{x}}$, where $\sigma_{\mathrm{x}}$ is the rms horizontal beam size ${ }^{4}$. This implies that $\mathrm{x}$-rays from the $6 \sigma_{\mathrm{x}}$ position of the electron beam will contain a background that is $\mathrm{e}^{-18}=1.5 \times 10^{-8}$ of the on-axis flux over the duration of the electron bunch. Thus, contributions to the background from the electron beam profile are not significant.

The dominant contribution to the long-pulse background comes from non-specular scattering from the beamline optics, and originates from surface roughness. The scattering is evaluated by measuring the transverse x-ray beam profile in the image plane of the beamline using a scanning slit pair as shown in Fig. 2. A Si(111) crystal is used to select monochromatic x-rays, and vertical slits are used to block the out-of-plan scattering which can further degrade the image quality.
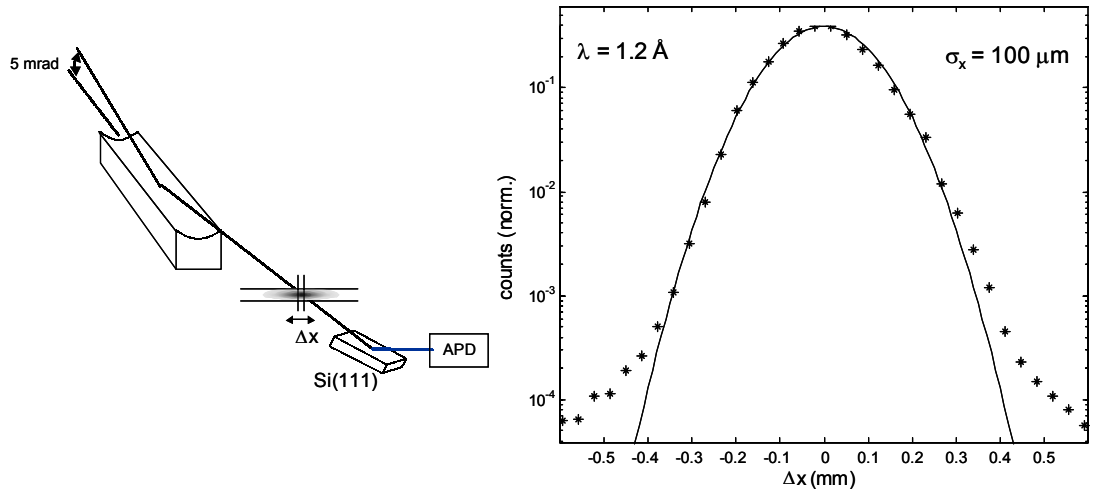

FIGURE 2. Measurement of horizontal x-ray beam profile at the image plane of ALS beamline 5.3.1. Monochromatic x-rays at $1.2 \AA$ are selected using a $\mathrm{Si}(111)$ crystal. Vertical slits are used to block out-ofplane scattering which degrades the image quality. The solid line is a normalized (area $=1$ ) Gaussian fit with $\sigma_{\mathrm{x}}=100 \mu \mathrm{m}$. 
Figure 2 shows measurements of the horizontal beam profile at a wavelength of $1.2 \AA$. The image follows a Gaussian profile with $\sigma_{\mathrm{x}}=100 \mu \mathrm{m}$, which is in good agreement with the predicted size of the electron beam: $\sigma_{\mathrm{x}}{ }^{2}=\beta_{\mathrm{x}} \varepsilon_{\mathrm{x}}+\left(\eta_{\mathrm{x}} \sigma_{\mathrm{E}}\right)^{2}$ where $\beta_{\mathrm{x}}=0.9 \mathrm{~m}, \varepsilon_{\mathrm{x}}=6.75 \mathrm{~nm} \cdot \mathrm{rad}$, and $\eta_{\mathrm{x}}=0.07 \mathrm{~m}$ are the horizontal $\beta$-function, emittance, and dispersion parameters respectively, and $\sigma_{\mathrm{E}}=10^{-3}$ is the normalized beam energy spread. The contributions from the non-specular scattering background become significant for $\Delta x>400 \mu \mathrm{m}\left(4 \sigma_{\mathrm{x}}\right)$, and are $\sim 10^{-4}$ at $\Delta \mathrm{x}=500 \mu \mathrm{m}$.

\section{Direct Measurement of Femtosecond X-rays}

Figure 3 shows a model calculation ${ }^{3}$ of the transverse differential profile (mod. laser on minus mod. laser off) of the electron beam for various values of the maximum energy modulation, $\Delta \mathrm{E}_{\max }$, relative to the nominal normalized beam energy spread, $\sigma_{\mathrm{E}}$. The calculation assumes a 75 fs laser pulse duration, and $75 \mathrm{ps}$ electron bunch duration (FWHM). The fraction of the total bunch charge, within the femtosecond slice, increases with increasing energy modulation (e.g. at $\Delta \mathrm{E} / \sigma_{\mathrm{E}}=6$ ), and then reaches a plateau at $\sim 10^{-4}$ of the total bunch charge. The expected transverse spatial profile of the femtosecond electron slice (and of the femtosecond x-rays) is derived from the differential electron beam vs. energy profile by a transformation of the horizontal scale given by $\Delta x=D_{x} \cdot \Delta E$, where $\Delta x$ is the horizontal displacement and $\mathrm{D}_{\mathrm{x}}=0.08 \mathrm{~m}$ is the dynamic dispersion at ALS bend magnet 5.3. For example, an energy modulation of $\Delta \mathrm{E} / \sigma_{\mathrm{E}}=6$ corresponds to a transverse displacement of $\Delta \mathrm{x}=480 \quad \mu \mathrm{m}$.

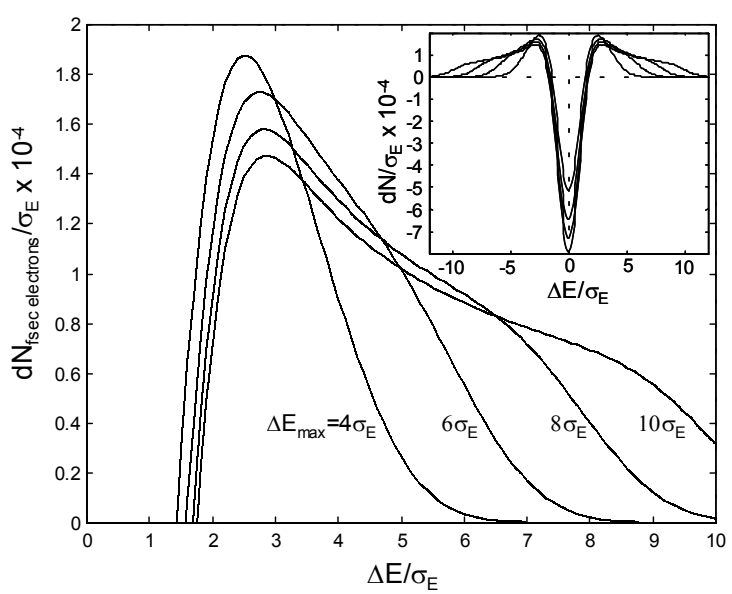

FIGURE 3. Calculated differential e-beam profiles showing the energy distribution of the femtosecond electron slice for maximum energy modulations of $\Delta \mathrm{E}_{\max }=4 \sigma_{\mathrm{E}}, 6 \sigma_{\mathrm{E}}, 8 \sigma_{\mathrm{E}}$, and $10 \sigma_{\mathrm{E}}$. Inset shows the full differential distribution, with increased electrons at higher (and lower) energies, and a loss of electrons at the nominal beam energy. The transverse distribution of the femtosecond $\mathrm{x}$-rays is identical, with a horizontal scale given by $\Delta x=D_{x} \cdot \Delta E$.

The transverse profile of the femtosecond x-rays is measured using the set-up shown in Fig. 2. Differential profile measurements are made with the modulating laser alternately on and off, in order to subtract the long-pulse background. A fixed vertical slit is used to block the out-of-plane scattering, and a horizontal slit is scanned to measure the femtosecond flux a various transverse positions, $\Delta \mathrm{x}$, from the beam center.

Figure 4 (left) shows the measured profile of the femtosecond $\mathrm{x}$-rays as a function of slit position, with a slit width of $50 \mu \mathrm{m}$. Femtosecond x-ray flux is observed at displacements as large as $700 \mu \mathrm{m}$, with the maximum flux at a displacement of $\sim 300 \mu \mathrm{m}$. The solid and dashed lines in Fig. 4 (left) are model calculations of the expected profile (as described above) assuming maximum energy modulations of $6 \sigma_{\mathrm{E}}, 7 \sigma_{\mathrm{E}}$, and $8 \sigma_{\mathrm{E}}$. The agreement with the measured profile indicates that the energy modulation of the electron bunch by the laser is in the range of $7 \sigma_{\mathrm{E}}(13.3$ $\mathrm{MeV}$ ). Figure 4 (right) shows the measured ratio of the femtosecond flux to the long-pulse background (integrating over a single bunch). A maximum signal/background ratio of nearly 1 is reached at $\Delta x=500 \mu \mathrm{m}$. This is in good agreement with the predicted femtosecond flux indicated in Fig. $3\left(\mathrm{dN} / \sigma_{\mathrm{E}} \sim 10^{-4}\right.$ at $\Delta \mathrm{E} / \sigma_{\mathrm{E}}=6$, and $\left.\Delta \mathrm{E}_{\max }=7 \sigma_{\mathrm{E}}\right)$ and the measured background $\left(\sim 10^{-4}\right)$ at $\Delta \mathrm{x}=500 \mu \mathrm{m}$ indicated in Fig. 2. Further improvement in the femtosecond flux to background ratio may be achieved by stronger modulation of the electron beam, thereby providing femtosecond flux at larger displacements, $\Delta \mathrm{x}$, where the background is lower. In addition, several approaches are being pursued to reduce the non-specular scattering from the mirror, which is the dominant contribution to the background. 

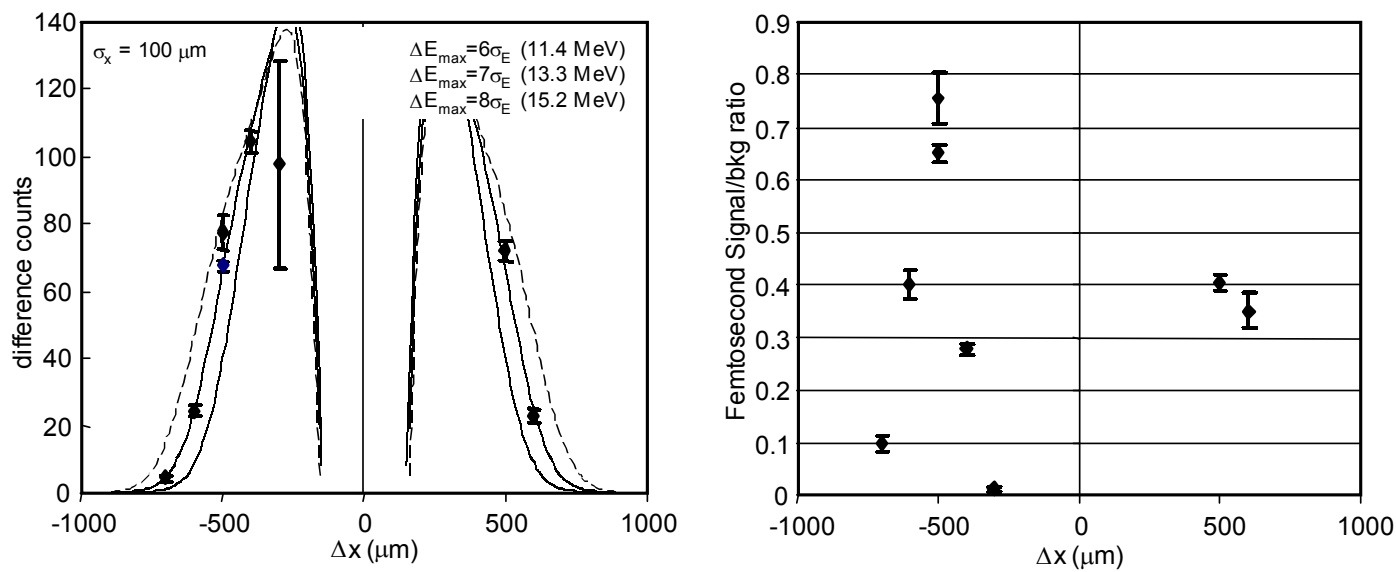

FIGURE 4. Left: measured horizontal distribution of femtosecond $x$-rays at $1.2 \AA$ as a function of slit position. Solid and dashed lines are fits from the predicted differential $\mathrm{x}$-ray profile assuming maximum electron energy modulation of $6 \sigma_{\mathrm{E}}(11.4$ $\mathrm{MeV}), 7 \sigma_{\mathrm{E}}(13.3 \mathrm{MeV})$, and $8 \sigma_{\mathrm{E}}(15.2 \mathrm{MeV})$. Right: measured ratio of femtosecond signal to long-pulse background, $\mathrm{F}$, as a function of slit position.

Figure 5 shows a direct measurement of the femtosecond synchrotron pulse duration at ALS beamline 5.3.1. This measurement is made by cross-correlating the visible light from the bend magnet with a delayed femtosecond pulse from the laser system using sum frequency generation in a non-linear optical crystal (BBO). The measurement indicates not only the duration of the synchrotron radiation, but the overall measurement resolution of the system since the same pump-probe set-up and delayed laser pulse is used for time-resolved experiments.

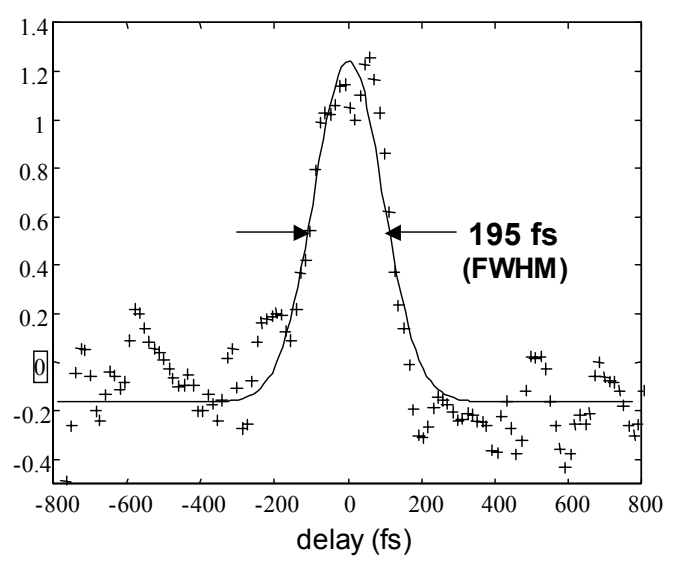

Figure 5. Cross-correlation measurement of visible femtosecond synchrotron radiation from ALS beamline 5.3.1.

\section{ACKNOWLEDGMENTS}

This work was supported by the U.S. Department of Energy, Office of Science, under Contract No. DEAC03-76SF00098. We gratefully acknowledge helpful discussions and advice from H.A. Padmore, and the technical assistance from ALS Accelerator Physics Group, ALS Engineering Group and ALS operations staff.

\section{REFERENCES}

1. Zholents, A.A. and Zolotorev, M.S., Phys. Rev. Lett., 76, 912-915, (1996).

2. Schoenlein, R.W., Chattopadhyay, S., Chong, H.H.W., Glover, T.E., Heimann, P.A., Shank, C.V., Zholents, A., and Zolotorev, M., Science, 287, 2237-2240, (2000).

3. Schoenlein, R.W., Chattopadhyay, S., Chong, H.H.W., Glover, T.E., Heimann, P.A., Shank, C.V., Zholents, A., and Zolotorev, M, Applied. Phys. B, 71, 1, (2000).

4. Zholents, A., and Decking, W., LBNL Report No. 45392, 1999. 